\title{
Untersuchungen zur Zoogeographie der Euphausiaceen (Crustacea) des Roten Meeres
}

\author{
R. WEIGMANN \\ Institut für Meereskunde an der Universität Kiel; Kiel, \\ und \\ Biologische Anstalt Helgoland*; Hamburg 50, Bundesrepublik Deutschland
}

\begin{abstract}
Studies on the zoogeography of the Euphausiacea (Crustacea) of the Red Sea. A joint programme on "Biota of the Red Sea and the Eastern Mediterranean" has been carried out by the Hebrew University of Jerusalem and the Smithsonian Institution of Washington between 1967 and 1972 in order to study the role played by the Suez Canal as a route for faunal exchanges between the Red Sea and Eastern Mediterranean. The euphausiids of this material have been examined and additional samples of zooplankton have been taken in the Gulf of Elat (Aqaba) in 1973 in order to study the vertical distribution of the euphausiids. Four species have been found to occur in the Northern Red Sea and the Gulf of Elat: Euphausia diomedeae, E. sanzoi, Stylocheiron affine and S. abbreviatum. Seven species were found in the Eastern Mediterranean of which Euphausia brevis is the dominant species. The present studies revealed that an exchange of Euphausiacea through the Suez Canal does not take place. The species of the Red Sea belong to the Indopacific fauna. Despite the particular historic-geographical and ecological situation of the Red Sea, no endemisms or subspecies have evolved there within the Euphausiacea.
\end{abstract}

\section{EINLEITUNG}

Seit jeher war das Rote Meer ein Anziehungspunkt für biologische Untersuchungen. Nach der Eröffnung des Suezkanals im Jahre 1869 verkürzte sich der Schiffahrtsweg, und erstmals gelangten danach auch Forschungsschiffe in dieses Meer. Trotz vieler seither von verschiedenen Nationen dorthin durchgeführter Expeditionen sind unsere Kenntnisse des Planktons, speziell des größeren Zooplanktons, in bezug auf Fragen der Verbreitung, Okologie und Physiologie der einzelnen Arten unzureichend.

Aus der Fülle der sich anbietenden Fragestellungen soll im folgenden vor allem auf zoogeographische Gesichtspunkte eingegangen werden, die sich aus der besonderen geographischen Lage des Roten Meeres zwischen Indischem Ozean und Mittelmeer ergeben, mit dem es durch den Suezkanal verbunden ist. Im Vordergrund wird die Frage stehen, ob die pelagische Fauna des Roten Meeres mehr indo-pazifischen oder mehr atlanto-mediterranen Charakter hat.

* Mitglied der Taxonomischen Arbeitsgruppe an der Biologischen Anstalt Helgoland. 
Die Untersuchungen dazu sind mit Euphausiaceen durchgeführt worden, die aus eigenen Zooplanktonfängen im Jahre 1973 stammen und aus dem Material der International Indian Ocean Expedition 1964/65 sowie solchem, das im Rahmen des Forschungsvorhabens „Biota of the Red Sea and the Eastern Mediterranean" gesammelt wurde. Zusätzlich werden die vorliegenden Arbeiten über Euphausiaceen von Torelli (1934), Tattersall (1939), Ponomareva (1968), Wiebe \& d'Abramo (1972) und Casanova (1974) zoogeographisch ausgewertet.

\section{MATERIAL UND METHODIK}

Das bearbeitete Material entstammt verschiedenen Untersuchungszeiträumen und Programmen.

(a) "Biota of the Red Sea and the Eastern Mediterranean". Dieses Programm wurde gemeinsam von der Hebrew University in Jerusalem und der Smithsonian Institution in Washington von 1967-1972 durchgefuhrt, um die Rolle des Suezkanals als Verbreitungsweg von Lebewesen zwischen Rotem Meer und Mittelmeer zu studieren. Die Arbeiten im Golf von Elat und im nördlichen Roten Meer wurden von den oben genannten Institutionen im August 1968 von dem äthiopischen Trawler „Menelik II" aus durchgeführt. Die Probennahme erfolgte mit einem modifizierten 6-Fuß-IsaacsKidd-Midwatertrawl bis zu $560 \mathrm{~m}$ Wassertiefe (Aron et al. 1965, Aron \& GoodYeAr 1969). Von diesem Material standen 16 Proben zur Verfügung; die Lage der Stationen ist Abbildung $1 \mathrm{zu}$ entnehmen.

Die Fänge im östlichen Mittelmeer erfolgten mit Planktonnetzen. Bei der Stationsbezeichnung der Proben folgte ich Por et al. (1972). Die in diesem Gebiet gefundenen Arten werden nicht quantitativ bewertet.

(b) In der Zeit von März bis Mai 1973 konnten von mir mehrere Ausfahrten in den Golf von Elat durchgeführt werden, um ergänzende Angaben zur Verteilung der Euphausiaceen zu erhalten. Der Ort der Probennahme lag bei Taba im inneren Teil des Golfes. Es wurden 91 Schließnetzfänge ( $\phi$ der Einströmöffnung $60 \mathrm{~cm}$, Maschenweite des Netzes $350 \mu$ ) in den Tiefen $25 \mathrm{~m}, 75 \mathrm{~m}, 100 \mathrm{~m}, 200 \mathrm{~m}, 300 \mathrm{~m}$ und $400 \mathrm{~m}$ zu verschiedenen Zeiten gemacht. Bei jeder Probennahme wurden $170 \mathrm{~m}^{3}$ Wasser filtriert.

\section{HYDROGRAPHIE DES UNTERSUCHUNGSGEBIETES}

Das Rote Meer ist - wie das Mittelmeer und das Schwarze Meer - ein in sich abgeschlossenes Nebenmeer mit einem ausgeprägten ariden Klima. Es ist im Norden durch den Suezkanal mit dem Mittelmeer und im Süden durch die flache Schwelle Babel-Mandeb mit dem Arabischen Meer verbunden. Die Temperatur im Roten Meer nimmt von Norden nach Süden hin zu ( $\mathrm{T}^{\circ} \mathrm{C}$ : von 21 auf $30^{\circ} \mathrm{C}$ ), der Salzgehalt in derselben Richtung ab ( $5 \%$ : von 41 auf $37 \%$ ). Das Wasser des Roten Meeres besitzt eine höhere Dichte $\left(\sigma_{t}=27,5\right)$ als das des angrenzenden Golfes von Aden $\left(\sigma_{t}=26,5\right)$ (Morcos 1970), was einen Ausstrom des Tiefenwassers über die Schwelle Bab-el-Mandeb in den Golf von Aden bewirkt. Das salzreichere Wasser sinkt auf die der jeweili- 


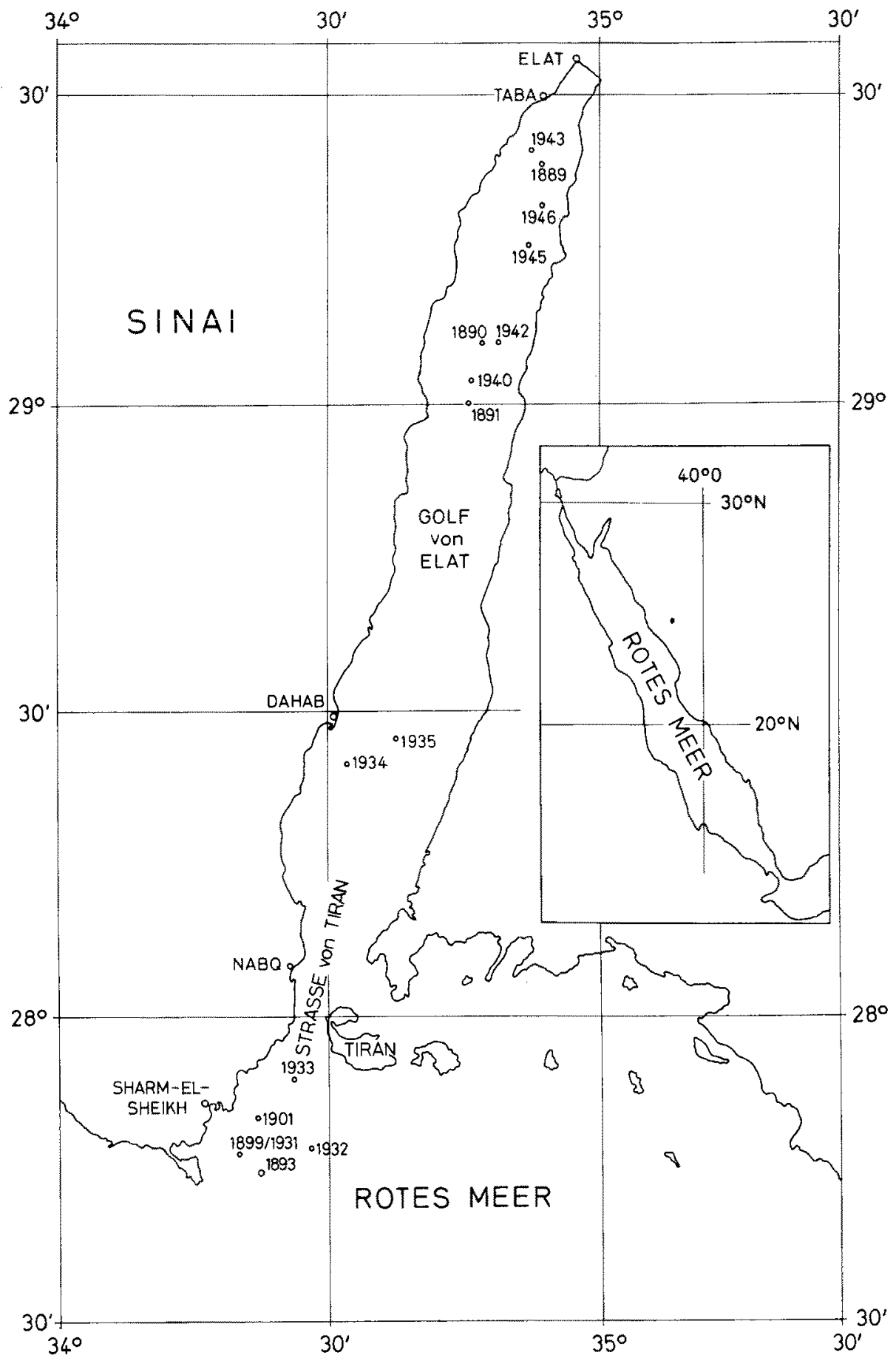

Abb. 1: Lage der Stationen des Programmes „Biota of the Red Sea and the Eastern Mediterranean" im August 1968, wovon die Euphausiaceen ausgewertet wurden 
gen Dichte entsprechenden Tiefen $a b$, um sich mit dem dort vorhandenen Wasser zu vermischen. An der Oberfläche strömt in umgekehrter Richtung das Wasser aus dem Golf von Aden in das Rote Meer ein, so daß das Wasser der tiefen Schichten des Golfes von Aden und des Arabischen Meeres im Roten Meer nicht vorhanden ist.

Im Norden besitzt das Rote Meer zwei Meeresarme mit unterschiedlichen hydrographischen Bedingungen. Der Golf von Suez ist ein sehr flaches Schelfgebiet (55 bis $73 \mathrm{~m}$ ), das praktisch nur Oberflächenwasser des Roten Meeres enthält. Die Oberflächentemperaturen liegen zwischen $21-24,5^{\circ} \mathrm{C}$, der Salzgehalt zwischen 40,4-42,5 S \% Der Golf von Elat (Aqaba) ist im Gegensatz dazu ein tiefes Becken ohne Schelf, mit Tiefen bis zu $1840 \mathrm{~m}$. Die Oberflächentemperatur des Wassers beträgt $21-23,5^{\circ} \mathrm{C}$, und auch in den größten Tiefen sinkt die Temperatur nicht unter $21^{\circ} \mathrm{C}$. Der Salzgehalt wird mit 40-41 S\% \% angegeben (Morcos 1970). Der Wasseraustausch zwischen dem Golf von Elat und dem Roten Meer erfolgt über den flachen Rücken der Straße von Tiran $(340 \mathrm{~m})$.

\section{ERGEBNISSE}

\section{"Biota of the Red Sea and the Eastern Mediterranean"}

Während dieses Programmes wurden im Golf von Elat und im nördlichen Roten Meer 16 Proben genommen. In dem gesamten Material wurden 4 Arten gefunden: Euphausia diomedeae, E. sanzoi, Stylocheiron affine und S. abbreviatum. Betrachtet man die Dominanz der Arten, so ist aus Tabelle $1 \mathrm{zu}$ ersehen, daß im Golf von Elat $S$. abbreviatum und $E$. diomedeae dominant sind, im nördlichen Roten Meer S. abbreviatum und E. sanzoi. S. affine ist im Golf von Elat regelmäßiger und in größerer Anzahl vorhanden als im nördlichen Roten Meer, wo sie seltener auftritt.

S. abbreviatum und $S$. affine sind epipelagische Arten und in allen drei Ozeanen im tropisch-subtropischen Bereich (von $40^{\circ} \mathrm{N}$ bis $40^{\circ} \mathrm{S}$ ) weit verbreitet. E. diomedeae ist ebenfalls eine epipelagische Art, die jedoch nur im Pazifischen und Indischen Ozean in den Breiten von $20^{\circ} \mathrm{N}$ bis $20^{\circ} \mathrm{S}$ vorkommt. E. sanzoi wurde bisher nur im Roten Meer und im Arabischen Meer gefunden. Diese epipelagische Art ist somit auf die genannten tropischen Gebiete beschränkt. Aus früheren Veröffentlichungen geht hervor, daß E. sanzoi meist nur spärlich in den Proben enthalten war (Torelli 1934, WeIGMANN 1970). In dem vorliegenden Material war sie in großer Menge vorhanden, vor allem geschlechtsreife $q \propto$ und $\delta \hat{\delta}$. Es kann nach diesen Befunden vermutet werden, daß E. sanzoi im nördlichen Roten Meer und im anschließenden Golf von Elat ihren Hauptlebensraum hat.

Im Rahmen desselben Programmes wurden auch 18 Proben im östlichen Mittelmeer, im Gebiet um Rhodos und Zypern sowie vor der israelischen und libanesischen Küste genommen. Sie enthielten 7 Arten (Tab. 2), von denen E. brevis regelmäßig in allen Proben vorhanden war und den Hauptbestand bildete. Die nächsthäufigen Arten waren E. krohnii, E. hemigibba, Thysanopoda subaequalis und Stylocbeiron submii; $S$. longicorne und $S$, abbreviatum traten nur selten in den Fängen auf. Alle hier identifizierten Arten leben epipelagisch, lediglich S. longicorne ist eine mesopelagische Art. Betrachtet man die thermische Affinität der Arten, so zeigt sich, daß nur E. krohnii 


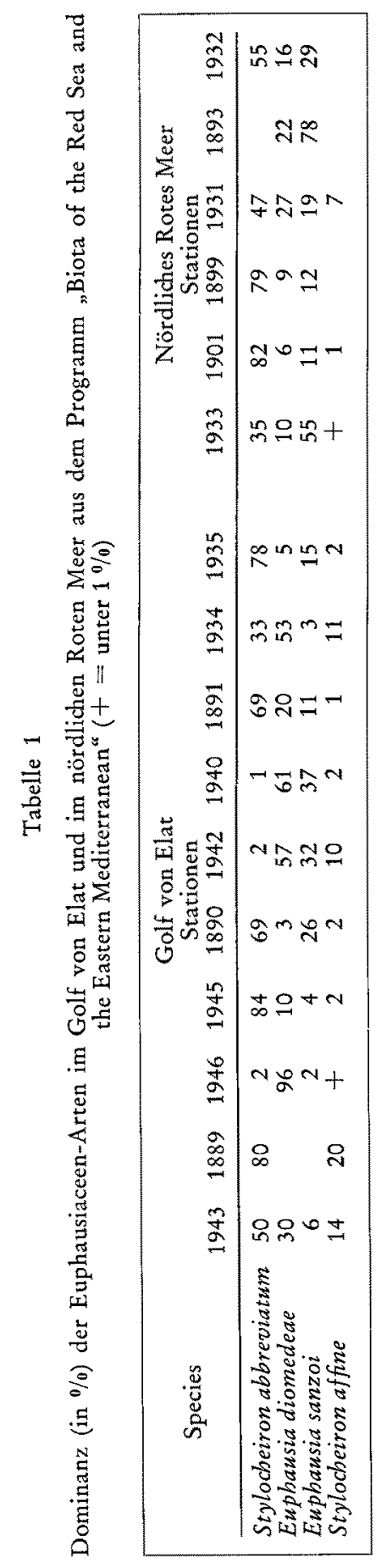


Tabelle 2

Die aus dem Programm "The Biota of the Red Sea and the Eastern Mediterranean" im östlichen Mittelmeer gefundenen Euphausiaceen-Arten. (Stationsfolge siehe Por et al. 1972)

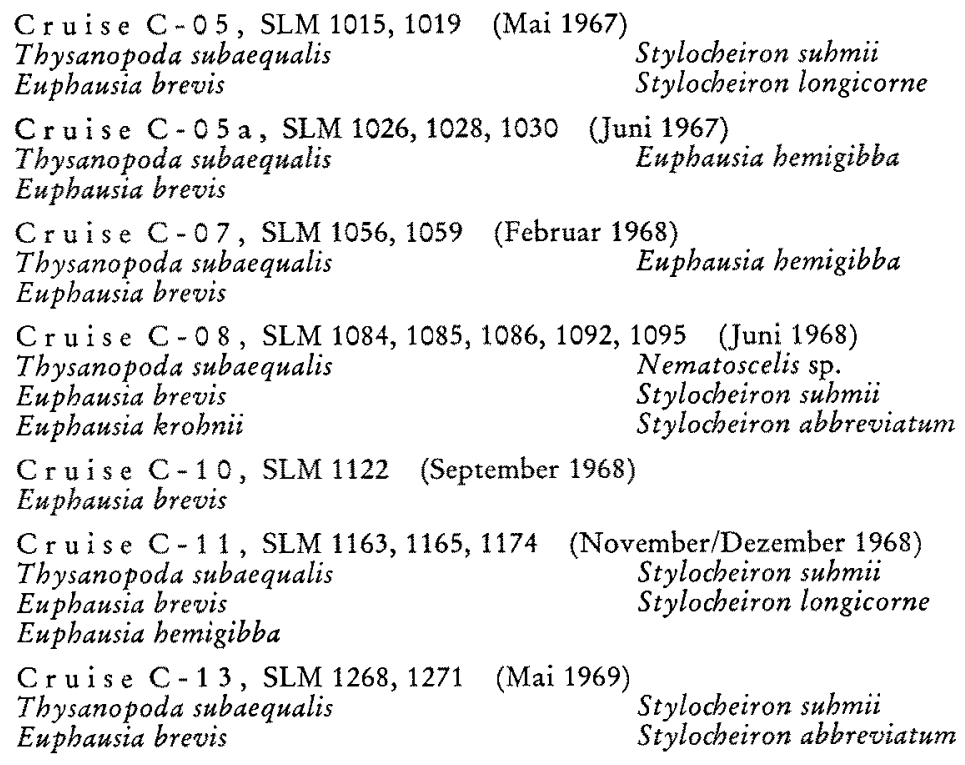

mehr in zentralen bis subarktischen Meeresgebieten lebt, während die übrigen in den subtropischen Lebensraum gehören (CASANOva-Soulier 1968).

\section{Zooplanktonfänge im Golf von Elat 1973}

Die größte Anzahl der Fänge wurde in den Tiefen $25 \mathrm{~m}, 75 \mathrm{~m}, 100 \mathrm{~m}$ und $200 \mathrm{~m}$ durchgeführt. Aus dem gesamten Material wurden vier Arten identifiziert: Euphausia diomedeae, E. sanzoi, Stylocheiron affine und S. abbreviatum; in der Hauptsache wurden aber nur Juvenile gefangen $(65 \%)$, die den einzelnen Arten zugeordnet werden konnten. E. sanzoi war nur unterhalb $100 \mathrm{~m}$ anzutreffen und im vorhandenen Material mit nur 17 Exemplaren vertreten. S. affine kam selten oberhalb von $75 \mathrm{~m}$ vor, darunter war diese Art zwar spärlich (zwischen 1-10 Tieren $/ 170 \mathrm{~m}^{3}$ ), aber sehr gleichmäßig in den Nachtzeiten verteilt. S. abbreviatum wurde niemals im 25-m-Horizont gefangen, in den übrigen Fängen der verschiedenen Tiefen wurden überwiegend Larven und Juvenile, selten adulte $q \phi$ und $\hat{\delta} \delta \hat{b}$ angetroffen. Die maximalen Ansammlungen wurden in der Zeit vor Mitternacht $\mathrm{z}$ wischen 100-300 $\mathrm{m}$ festgestellt.

E. diomedeae war die dominante Art, die stets in allen Proben angetroffen wurde. An Hand des Materials läßt sich ein ungefähres Bild der Vertikalverteilung dieser Art darstellen (Abb. 2). In der Zeit ab $18 \mathrm{Uhr}$ (Eintritt der Dunkelheit 18 bis 18.30 Uhr Ortszeit) erfolgt ein aktives Ansteigen der Tiere in die oberen Wasserschichten, wobei 


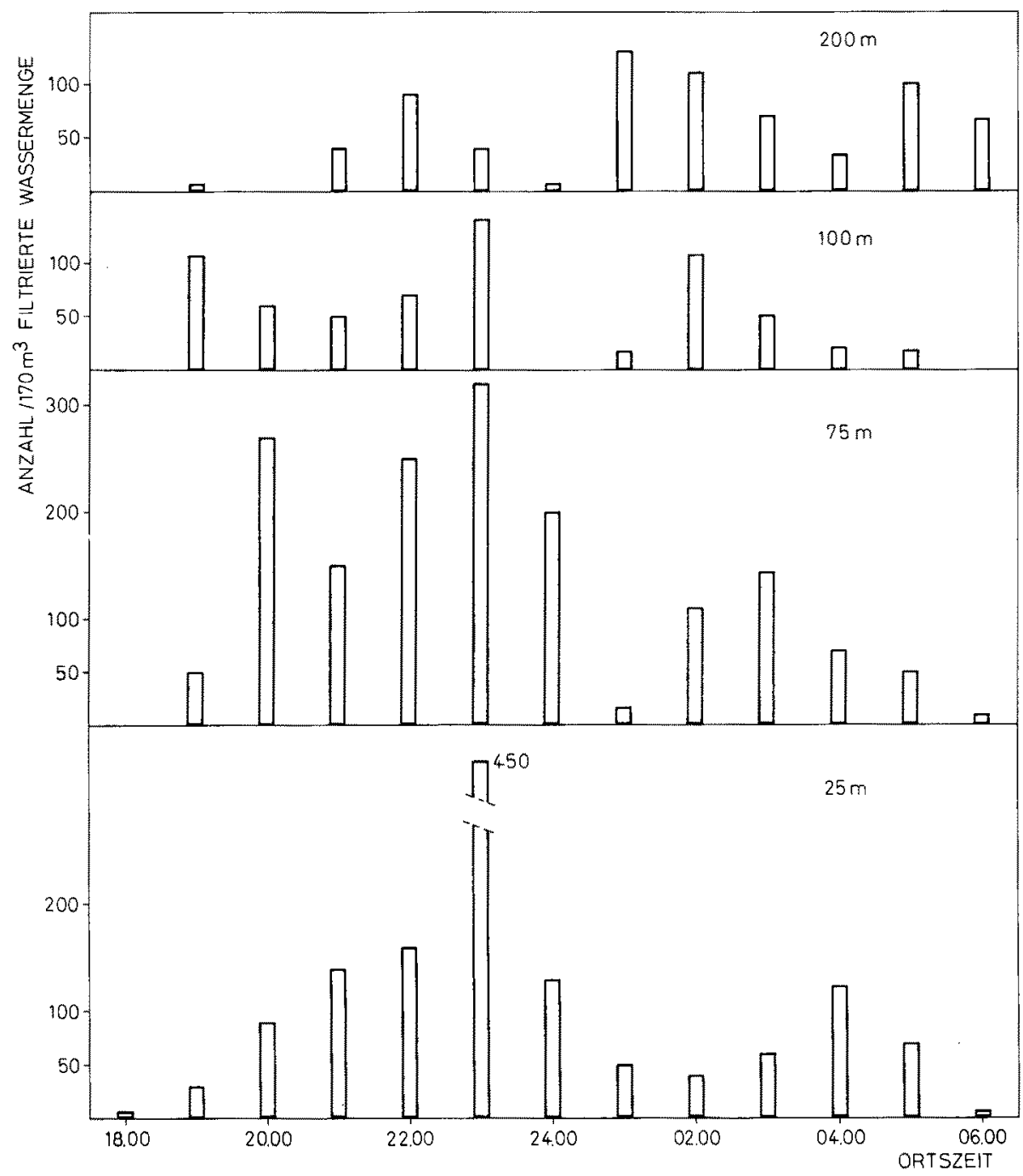

Abb. 2: Vertikalverteilung von Euphausia diomedeae im Golf von Elat. Die Angaben beruhen auf 5 Dauerstationen bei Taba

die maximalen Ansammlungen in der Zeit von 22-23 Uhr in den Tiefen $25 \mathrm{~m}$ und $75 \mathrm{~m}$ anzutreffen waren. Nach Mitternacht war $E$. diomedeae in allen befischten Schichten ohne nennenswerte Unterschiede verteilt. Gegen Morgen erfolgt dann das Absteigen in die tieferen Wasserschichten, so daß in der Zeit nach 6 Uhr keine Tiere mehr oberhalb $200 \mathrm{~m}$ Tiefe angetroffen wurden. $\mathrm{Zu}$ der dargestellten Vertikalverteilung muß hinzugefügt werden, daß im Golf von Elat keine thermischen Sprungschichten ausgebildet sind, sondern das Wasser eine gleichbleibende Temperatur von $21-22^{\circ} \mathrm{C}$ besitzt. 


\section{DISKUSSION}

\section{Artenzusammensetzung im Roten Meer}

Bei den Euphausiaceen kann man im Roten Meer eine deutlich unterschiedliche Zusammensetzung und Verbreitung der vorhandenen Arten feststellen. Die qualitative und quantitative Artenzusammensetzung ist im südlichen und im nördlichen Teil verschieden. Im südlichen Teil des Roten Meeres sind Euphausia diomedeae und E. distinguenda dominant, im nördlichen Teil $E$. sanzoi und Stylocheiron abbreviatum, die im Süden seltener sind. Einige Arten des südlichen Teiles ( $E$, distinguenda, $P$. latifrons) sind im nördlichen abwesend, jedoch haben wir unglücklicherweise keine exakten Angaben über den mittleren Teil des Roten Meeres, so daß zur Zeit kein vollständiges Bild der geographischen Artensukzession gegeben werden kann. Betrachtet man die Artenzusammensetzung im Golf von Elat und im nördlichen Roten Meer, so sieht man, daß in diesen Meeresgebieten dieselben Arten leben (Tab. 1). Es ist anzunehmen, daß die Straße von Tiran für diese Arten keine Barriere darstellt. Entsprechende Ergebnisse ergaben sich auch für die Verbreitung der Fische beiderseits dieser Meerenge (Aron \& GOODYEAR 1969).

Generell kann man feststellen, daß bei den Euphausiaceen die Zahl der Genera und Arten vom Arabischen Meer über das Rote Meer bis hin zum Golf von Elat abnimmt (Tab. 3). Im Arabischen Meer leben ca. 33 Arten (Tattersall 1939, PonomaReva 1964), im südlichen und mittleren Teil des Roten Meeres 10 (Ponomareva 1968, WEIGMANN 1970), im nördlichen Roten Meer und im Golf von Elat nur noch 4 Arten. Arten, die im Arabischen Meer sehr weit verbreitet sind, E. tenera und E. paragibba, fehlen völlig im Roten Meer. Weiterhin ist bemerkenswert, daß drei Genera, die meist meso- bis bathypelagische Arten enthalten - Thysanopoda, Nematobrachion und Nematoscelis - nicht im Roten Meer zu finden sind, obgleich sie im Arabischen Meer und im Golf von Aden eine weite Verbreitung haben. Die Erklärung für diese Tatsache scheint in der geringen Tiefe der Straße Bab-el-Mandeb $(100 \mathrm{~m})$ zu liegen. Die Euphausiaceen führen tägliche Vertikalwanderungen durch, wobei sie am Tage in $300-800 \mathrm{~m}$ Tiefe leben und des nachts in höhere Wasserschichten aufsteigen. Die oben erwähnten Gattungen steigen nachts selten höher als $200 \mathrm{~m}$. Es ist anzunehmen, daß die flache Schwelle ein Hinüberwandern ihrer Arten verhindert. Ein begrenzender ökologischer Faktor könnte die höhere Minimaltemperatur der tieferen Wasserschichten des Roten Meeres sein $\left(21,5-22,0^{\circ} \mathrm{C}\right)$, die ja eine spezielle Anpassung der Tiere erfordern würde.

Die zahlenmäßige Abnahme der Arten vom Arabischen Meer zum Roten Meer ist auch innerhalb anderer Zooplanktongruppen bekannt; so z. B. betont ALvarino (1972), daß die Anzahl der Arten bei den Siphonophoren im Roten Meer geringer ist als im Indischen Ozean und der Golf von Elat artenärmer als das Rote Meer. Bei den calanoiden Copepoden verringert sich die Artenzahl von 270 im Arabischen Meer auf $158 \mathrm{im}$ Roten Meer (KIMOR 1973). Die Reduzierung der Artenzahl ist auch bei den Amphipoden-Hyperiiden (Indischer Ozean: 35 Arten, Rotes Meer: 20 Arten, nach Walker 1909 und Spandl 1924), Chaetognathen (Arabisches Meer: 13 Arten, Golf von Elat: 5 Arten, nach Furnestin 1958, Furnestin \& Codaccioni 1968) und Appendicularien (Arabisches Meer: 19 Arten, Golf von Elat: 3 Arten, nach Fenaux 1960, 
1966) bekannt. Nach Angaben von Furnestin (1968) fehlen im Roten Meer ebenfalls meso- und bathypelagische Chaetognathen-Arten.

\section{Beziehungen zum Arabischen Meer}

Es stellt sich die Frage nach der Beziehung der Fauna des Roten Meeres zum benachbarten Arabischen Meer. Das Rote Meer hat als ein typisches Randmeer Verbindung zur atlantisch-mediterranen Fauna über den Suezkanal und über die Straße Babel-Mandeb zur indo-pazifischen. Aus Tabelle 3 kann man entnehmen, daß bei den Euphausiaceen die Artenzusammensetzung zwischen dem Roten Meer und dem Mittelmeer grundsätzlich verschieden ist, ausgenommen zwei Arten, Euphausia brevis und Stylocheiron abbreviatum. Diese beiden Arten leben aber ebenso im Arabischen Meer. Von den bislang 10 Arten, die für das Rote Meer identifiziert wurden, sind vier auf den indo-pazifischen Raum beschränkt: $E$. sanzoi, E. diomedeae, E. distinguenda und $P$. latifrons. Es ist wohl mit Sicherheit anzunehmen, daß diese von Süden her eingewandert sind. Die verbleibenden 6 Arten, die im Roten Meer leben, sind Kosmopoliten, die im tropisch-subtropischen Bereich aller drei Ozeane leben.

Tabelle 3

Euphausiaceen-Arten mit gemeinsamem Vorkommen im Golf von Elat, Roten Meer, Arabischen Meer und Mittelmeer nach Angaben von Torelli (1934), Tattersall (1939), Ponomareva (1968), WEIGMANN (1970), WIEBE \& D'ABRAMO (1972), Casanova (1974) und eigenen Ergebnissen

\begin{tabular}{|lcccc|}
\hline \multicolumn{1}{|c}{ Species } & $\begin{array}{c}\text { Golf von } \\
\text { Elat }\end{array}$ & Rotes Meer & $\begin{array}{c}\text { Arabisches } \\
\text { Meer }\end{array}$ & Mittelmeer \\
\hline Euphausia sanzoi & + & + & + & \\
Euphausia diomedeae & + & + & + & \\
Stylocheiron affine & + & + & + & + \\
Stylocheiron abbreviatum & + & + & + & + \\
Euphausia distinguenda & & + & + & \\
Pseudeuphausia latifrons & & + & + & \\
Euphausia mutica & & + & + & + \\
Euphausia brevis & & + & + & + \\
Stylocheiron carinatum & & + & + & + \\
Stylocheiron elongatum & & & + & + \\
Thysanopoda subaequalis & & & + & + \\
Nematoscelis atlantica & & & + & + \\
Stylocheiron submii & & & + \\
Stylocheiron longicorne & & & + \\
Stylocheiron maximum & & & + \\
Euphausia bemigibba & & & + \\
Euphausia krobnii & & & & + \\
Meganyctiphanes norvegica & & & & + \\
Nematoscelis megalops & & & & + \\
\hline
\end{tabular}

Auch für die Fischwelt verneinte GoHar (1955) Gemeinsamkeiten zwischen dem Roten Meer und dem Mittelmeer; er nimmt an, daß die heutige Fischfauna rein indopazifischen Ursprungs ist. Bemerkenswert ist bei einem Vergleich der Fauna des Roten 
Tabelle 4

Bekannte Euphausiaceen-Arten des Roten Meeres und Arten mit zweifelhafter Validität*

\begin{tabular}{|c|c|c|c|c|c|}
\hline Species & $\begin{array}{c}\text { Tokelli } \\
(1934)\end{array}$ & $\begin{array}{c}\text { PonOMAREVA } \\
(1968)\end{array}$ & $\begin{array}{l}\text { WEIGMANN } \\
(1970)\end{array}$ & $\begin{array}{c}\text { CASANOVA } \\
(1974)\end{array}$ & $\begin{array}{l}\text { WEIGMANN } \\
(1974)\end{array}$ \\
\hline Eupbausia diomedeae & + & + & + & + & + \\
\hline Stylocbeiron affine & + & + & + & + & + \\
\hline Euphausia sanzoi & + & + & + & & + \\
\hline Euphansia distinguenda & + & + & + & + & \\
\hline Stylocheiron abbreviatum & & + & + & t & + \\
\hline Pseudeuphansia latifrons & + & & + & & \\
\hline Stylocbeiron carinatum & & + & & + & \\
\hline Stylocheiron submii & & & & + & \\
\hline Euphausia brevis & & + & & & \\
\hline Euphausia mutica & & + & & & \\
\hline Stylocheiron elongatum & & + & & & \\
\hline "Euphausia messanensis & + & & & & \\
\hline *Pseudeuphausia colosii & + & + & & & \\
\hline "Stylocheiron armatum & + & & & & \\
\hline
\end{tabular}

Meeres mit der des Indischen Ozeans jedoch, daß nach Klausewitz (1964) „trotz sehr vieler Gemeinsamkeiten und des nicht zu leugnenden indo-pazifischen Ursprungs eine Reihe bemerkenswerter Unterschiede, als Endemismen, Vikarianten, Subspezies und andere abweichende Formen" innerhalb der Gruppe der Fische zu finden sind.

Innerhalb der Euphausiaceen sind keine von den Originalbeschreibungen abweichenden Formen gefunden worden, d. h, nach meinen Befunden fehlen Endemismen oder Unterarten im gesamten Roten Meer und im Golf von Elat. Eine mögliche Erklärung wäre, daß sich die Euphausiaceen als eine konservative Gruppe phylogenetisch sehr langsam entwickeln und der Zeitraum (600000 Jahre seit dem Günz-MindelInterglazial), der der Neubesiedlung des Roten Meeres von Süden her zur Verfügung stand, zur Bildung neuer Arten nicht ausgereicht hat. Wahrscheinlich ist die Isolation dieses Randmeeres nicht groß genug, und es sind zu wenig ökologische Nischen vorhanden. Das Rote Meer ist zwar ein extremer, jedoch stabiler Biotop, auch deshalb könnte die Evolutionsgeschwindigkeit bei den Populationen der vorhandenen Euphausiaceen-Arten verlangsamt sein.

Der Suezkanal - eine Verbindung zwischen Mittelmeer und Rotem Meer

Die Rolle des Suezkanals als Verbindung zwischen zwei sehr unterschiedlichen marinen Regionen - der mediterranen und der erythräischen - ist schon oft diskutiert worden. Hier nun soll untersucht werden, ob der Suezkanal für die holoplanktischen Euphausiaceen ein möglicher Wanderweg ist oder eine Verbreitungsschranke darstellt. Nach Angaben von Casanova (1974) leben schon im Golf von Suez keine Euphausiaceen mehr, da die geringe Tiefe (ca. $50 \mathrm{~m}$ ) und die sehr hohe Salinität (41-42,5 S \%oo) keine günstigen Umweltbedingungen darstellen. Die Tiefe des Suezkanals mit 12 bis 
$15 \mathrm{~m}$, der noch höhere Salzgehalt von $45 \mathrm{~S} \%$ und die Temperatur, die leicht bis $25^{\circ} \mathrm{C}$ ansteigen kann, stellen extreme Lebensbedingungen dar, die nur euryöke Arten vertragen. Für die im Roten Meer vorkommenden Euphausiaceen-Arten als Vertikalwanderer mit großen Anforderungen an tiefe Wasserschichten kann eine Wanderung durch den Suezkanal ausgeschlossen werden. Für eine eventuelle Wanderung kämen nur neritische Arten in Frage, die jedoch bisher weder im Golf von Elat noch im Golf von Suez oder im nördlichen Roten Meer gefunden wurden.

Es liegen nur wenig Informationen über den Austausch von holoplanktischen Organismen zwischen dem Mittelmeer und dem Roten Meer durch den Suezkanal vor; so sind unter anderem vermutlich zwei calanoide Copepoden-Arten vom Roten Meer ins Mittelmeer eingewandert (Berdugo 1968) und eine Appendicularien-Art vom Mittelmeer ins Rote Meer (FEnaux 1960). Alvarino (1972) fand unter den Siphonophoren zwei Arten, die durch den Suezkanal in beiden Richtungen gewandert sein könnten. Jedoch sind diese Arten kosmopolitisch verbreitet ,and the source of the populations in the regions adjacent to the Suez Canal may be in the adjacent oceanic regions". Die Chaetognathen-Fauna des östlichen Mittelmeeres erweist sich nach Untersuchungen von FurNestin \& Baranca (1968) als sehr unterschiedlich zu der des Roten Meeres und des Golfes von Elat, und es scheint, daß in diesem Fall der Suezkanal eher eine Barriere darstellt als einen möglichen Verbindungsweg.

In vielen systematischen Gruppen findet ein Artenaustausch durch den Suezkanal statt, z. B. Ostracoden, Amphipoden, decapode Crustaceen, Mollusken, Polychaeten, Fische und auch Algen (STEINITZ 1967, Krmor 1972). Besonders für die Fische wird ein aktiver Austausch in beiden Richtungen beschrieben (GoHAR 1955, KLAUSEwITZ 1960, 1964, Por 1971, Steinitz \& Ben-Tuvia 1972), wobei aber eine größere Anzahl von Arten (26) vom Roten Meer durch den Suezkanal ins östliche Mittelmeer eingewandert ist als in entgegengesetzter Richtung (8 Arten) (Ben-Tuvia 1966). Bei den Fisch-Immigranten aus dem Roten Meer handelt es sich generell um neritische Küstenwasserarten.

Man kann annehmen, daß im Suezkanal keine holoplanktischen Gruppen wie Chaetognathen und Euphausiaceen leben, die in allen Entwicklungsstadien große Ansprïche an die Wassertiefe stellen. In diesen Gruppen kommt es nicht zum Austausch von Arten durch den Suezkanal - er erweist sich in diesen Fällen als Verbreitungsschranke.

\section{ZUSAMMENFASSUNG}

1. An Hand von Material, das aus dem Arbeitsprogramm „Biota of the Red Sea and the Eastern Mediterranean" stammt, wurde die Artenzusammensetzung der Euphausiaceen im nördlichen Roten Meer, im Golf von Elat (Aqaba) und im östlichen Mittelmeer untersucht.

2. Für das nördliche Rote Meer und den Golf von Elat, die artenidentisch sind, wurden vier Arten bestimmt: Euphausia diomedea, E. sanzoi, Stylocheiron affine und $S$. abbreviatum.

3. Aus dem östlichen Mittelmeer konnten sieben Arten identifiziert werden, wovon $E$. brevis den Hauptbestand bildete. Die Artenzusammensetzung in diesem Gebiet war eine völlig andere als im Roten Meer. 
4. Nach den vorliegenden Ergebnissen wird ein Austausch von Euphausiaceen durch den Suezkanal ausgeschlossen; die im Roten Meer lebenden Arten gehören zum indo-pazifischen, die im Mittelmeer lebenden Arten zum atlanto-mediterranen Faunenbereich.

5. Trotz der besonderen historisch-geographischen und ökologischen Situation des Roten Meeres ist es innerhalb der Euphausiaceen nicht zur Bildung von Endemismen oder Unterarten gekommen.

Danksagungen. Für die Bereitstellung des Materials und die notwendige Hilfe möchte ich Herrn Prof. Dr. D. Por (Jerusalem), Herrn Prof. Dr. B. Kimor (Haifa), Fräulein I. Ferber und G. Gorsky (Jerusalem) danken. Mein besonderer Dank gilt allen Mitarbeitern des Marine Biological Laboratory in Elat, auf deren Hilfe ich angewiesen war und die mir jederzeit gewährt wurde. Die Arbeit wurde mit finanzieller Unterstützung der Deutschen Forschungsgemeinschaft durchgeführt.

\section{ZITIERTE LITERATUR}

Alvarino, A., 1972. Distribution of siphonophores in the regions adjacent to the Suez and Panama Canals. Congr. int. Zool. 17, 1-30 (Preprint).

ARON, W. \& GOODYEAR, R. H., 1969. Fishes collected during a mid-water trawling survey of the Gulf of Elat and the Red Sea. Israel J. Zool. 18, 237-244.

- Ahlstrom, E. H., Bary, B. M., Be, A. W. H. \& Clarke, W. D., 1965. Towing characteristics of plankton sampling gear. Limnol. Oceanogr. 10, 333-340.

Ben-Tuvia, A., 1966. Red Sea fishes recently found in the Mediterranean. Copeia 1966, 254275.

Berdugo, V., 1968. Sur la présence dans la Méditerranée orientale de 2 espèce du genre Calanopia (Copepoda; Calanoida). Rapp. P.-v. Réun. Commn int. Explor. scient. Mer Méditerr. 18, 445-446.

Casanova-Sovlier, B., 1968. Les Euphausiacés de la Méditerranée. Congr. Commn Explor. scient. Mer Méditerr. 21, 1-62.

Casanova, B., 1974. Les Euphauisiacés de Méditerranée. Thèse de I'Université de Provence Aix-Marseille I. 380 pp.

FENAUX, R., 1960. Sur quelques appendiculaires d'Israel. Bull. Sea Fish. Res. Stn Israel 29, 3-7.

- 1966. Les apendiculaires de la Mer Rouge (note faunistique). Bull. Mus. natn Hist. nat., Paris 2 (38), 784-785.

Furnfstin, M. L., 1958. Contributions to the knowledge of the Red Sea. - Quelques échantilons de zooplancton du Golfe d'Eylath (Akaba). Bull. Sea Fish. Res. Stn Israel 16, 6-14.

- \& Balanca, J., 1968. Chaetognathes de la Mer Rouge Archipel Dahlac. Bull. Sea Fish. Res. Stn Israel 52, 3-20.

- \& CodaccronI, J. C., 1968. Chaetognathes du NW de l'Océan Indien (golfe d'Aden-mer d'Arabie-golfe d'Oman-golf persique). Cah. O. R. S. T. O. M. (Océanogr.) 6, 143-171.

Gohar, H. A. F., 1955. The place of the Red Sea between the Indian Ocean and the Mediterranean. Hidrobiol. Istanb. 2, 47-82.

KImor, B., 1972. The Suez Canal as a link and a barrier in the migration of planktonic organisms. Israel J. Zool. 21, 391-403.

KLAUSEWITZ, W., 1960. Systematisch-evolutive Untersuchungen über die Abstammung einiger Fische des Roten Meeres. Zool. Anz. (Suppl) 23, 175-182.

- 1964. Die Erforschung der Ichthyofauna des Roten Meeres. In: Klunzinger, C. B.: Synopsis der Fische des Roten Meeres. Cramer, Weinheim, Getr. Pag. (Reprint).

Morcos, S. A., 1970. Physical and chemical oceanography of the Red Sea. Oceanogr. mar. Biol. 8, 73-202. 
Ponomareva, L. A., 1964. On the Euphausiacea of the Arabian Sea and the Bay of Bengal. Trudy Inst. Okeanol. 64, 265-270.

- 1968. Euphausiids of the Red Sea collected in summer 1966 by R. V. "Academian S. Vavilov". Mar. Biol. 1, 263-265.

Por, F. D., 1971. One hundred years of Suez Canal - a century of lessepian migration: retrospect and viewpoints. Syst. Zool. 20, 138-159.

- Steinitz, H. \& Ferber, I., 1972. The biota of the Red Sea and the Eastern Mediterranean (1967-1972): A survey of the marine life of Israel and surroundings. Israel J. Zool. 21, 459-523.

SpAndr, H., 1924. Die Amphipoden des Roten Meeres der Expedition mit S. M. Schiff "Pola" in das Rote Meer 1895-98. Denkschr. Akad. Wiss., Wien 49, 19-73.

Steinitz, H., 1967. A tentative list of immigrants via the Suez Canal. Israel J. Zool. 16, $166-169$.

- \& Ben-Tuvia, A., 1972. Fishes of the Suez Canal. Israel J. Zool. 21, 385-389.

Tattersall, W. M., 1939. The Euphausiacea and Mysidacea of the John Murrey Expedition to the Indian Ocean. Scient. Rep. John Murrey Exped. 5 (8), 203-246.

Torelli, B., 1934. Eufausiacei del Mar Rosso. Memorie R. Com. talassogr. ital. 208, 1-17.

Walker, A. O., 1909. Amphipoda Hyperiidea of the "Sealark" Expedition to the Indian Ocean. Trans. Linn. Soc. Lond. (Zool.) 13, 49-55.

WEIGMANN, R., 1970. Zur OKkologie und Ernährungsbiologie der Euphausiaceen (Crustacea) im Arabischen Meer. Meteor-ForschErgeba. D 5, 11-52.

WIEBE, P. H. \& D'ABRAMO, L. D., 1972. Distribution of euphausiid assamblages in the Mediterranean Sea. Mar. Biol. 15, 139-149.

Anschrift der Autorin: Dr. Renate Weigmann

Institut für Meereskunde

an der Universität Kiel

23 Kiel

Düsternbrooker Weg 20

Bundesrepublik Deutschland 